Psychiatria clin. 1981;14:66

\title{
Nachruf auf Professor H. Mitsuda
}

D. Der Herausgeber dieser Zeitschrift hat die traurige Pflicht des Todes des langjährigen Mitherausgebers der „Psychiatria Clinica” Prof. Hisatoshi Mitsuda (15. Juni 1910- 14. Juli 1979)zugedenken.Prof.Mfsw < áwareinerderinEuropa am besten bekannten japanischen Psychiater der schon früh in seinen eigenen Arbeiten Themen aufgenommen hat die nach wie vor wichtige und die praktische Psychiatrie in hohem Masse beeinflussende Themen der wissenschaftlichen Psy-chiatrie sind. Zu erinnern ist vor allem an die Bemühungen von Mitsuda um eine klinische Genetik und ihre Anwendung zur Lösung offener Probleme bezüglich der Gruppe der Schizophrenien und der sogenannten atypischen Psychosen. Erfreulicherweise sind die Gedanken und Arbeiten von Mitsuda in repräsentativen englischsprachigen Publikationen leicht zugänglich so in den drei von ihm allein oder zusammen mit seinem langjährigen wissenschaftlichen Mitarbeiter Fukuda herausgegebenen Bänden „Clinical Genetics in Psychiatry” (1967) ", Biological Mechanisms of Schizophrenia and Schizophrenia-like Psychoses" (1974) und ," World Issues in the Problems of Schizophrenic Psychoses" (1979). Die originellen Beiträge von Mitsuda zu unserer Wissenschaft werden dafür sorgen dass sein Andenken lebendig bleibt! P. Berner 